\title{
Eukaryotes learn how to count: quorum sensing by yeast
}

\author{
George F. Sprague Jr. ${ }^{1,3}$ and Stephen C. Winans ${ }^{2}$ \\ ${ }^{1}$ Institute of Molecular Biology, Department of Biology, University of Oregon, Eugene, Oregon 97403, USA; ${ }^{2}$ Department of \\ Microbiology, Cornell University, Ithaca, New York 14853, USA
}

Signaling mechanisms that govern physiological and morphological responses to changes in cell density are common in bacteria. Quorum sensing, as such signal transduction processes are called, involves the production of, release of, and response to hormone-like molecules (autoinducers) that accumulate in the external environment as the cell population grows. Quorum sensing is found in a wide variety of bacteria, both Gram positive and Gram negative, and the spectrum of physiological functions that can be regulated is impressive indeed. Variation in the nature of the extracellular signal, in the signal detection machinery, and in the mechanisms of signal transmission demonstrates the evolutionary adaptability of quorum sensing systems for multiple uses. One testament to the range of these uses and to the general importance of quorum sensing in bacterial physiology comes from a PubMed search using "quorum" as the query: Well over 1000 articles have been published in the last 10 years. Here, we review the universal strategies that characterize bacterial quorum sensing and provide a glimpse of the diverse physiological settings in which it operates. We then discuss recent evidence for quorum sensing in different fungal species and conclude with an especially compelling case in the budding yeast Saccharomyces cerevisiae, described by Chen and Fink (2006) in this issue of Genes \& Development.

\section{Quorum sensing: the bacterial paradigm}

Many groups of bacteria exhibit complex patterns of coordinated behavior, and diffusible chemical signals have been either demonstrated or suspected to coordinate these activities. In the past $15 \mathrm{yr}$, scientists have documented a rich lexicon of signaling systems in many groups of microorganisms (for review, see Pappas et al. 2004). These chemical signals, sometimes referred to as autoinducers, are essentially bacterial pheromones and are required for diverse behaviors, including biolumines-

${ }^{3}$ Corresponding author.

E-MAIL gsprague@molbio.uoregon.edu; FAX (541) 346-4854.

Article and publication are at http://www.genesdev.org/cgi/doi/10.1101/ gad.1432906. cence, the horizontal transfer of DNA, the formation of biofilms, and the production of pathogenic factors, antibiotics, and other secondary metabolites (Whitehead et al. 2001). Intercellular signaling is often thought to provide a means to estimate population densities, hence the term "quorum sensing" (Fuqua et al. 1994). According to this idea, bacteria can estimate their numbers by releasing and detecting a particular chemical signal. However, these molecules could also allow the detection of a diffusion barrier (Redfield 2001). Supporting this idea, single cells of Staphylococcus aureus can induce quorum-dependent genes when confined within a host endosome (Qazi et al. 2001). Most likely, accumulation of these signals is facilitated both by a quorum of bacteria and by a diffusion barrier.

\section{Gram-positive bacteria}

Most of the signaling systems found in Gram-positive bacteria use oligopeptides as signals that are detected by two-component phosphorelay proteins (Dunny and Leonard 1997). In many cases, the receptor is a membranespanning histidine kinase, while in other systems the receptor is a cytoplasmic phosphatase that dephosphorylates a response regulator. A third type of peptide receptor, found in Enterococcus faecalis, is a transcriptional repressor (Shi et al. 2005). However, in contrast to the common use of peptide signals, one group of Gram-positive bacteria, the Actinomycetes, uses $\gamma$-butyrolactone signals to regulate the production of antibiotics and other secondary metabolites (Natsume et al. 2004).

Several peptide-dependent signaling systems in Grampositive organisms have been intensively studied. For example, the agrBDCA locus of $S$. aureus regulates a battery of pathogenesis genes via a peptide derived from AgrD. AgrD is processed and exported by AgrB, and detected by the AgrA/AgrC two-component system (Fig. $1 \mathrm{~A}$; Lyon and Novick 2004). AgrD is synthesized as a 45 -amino-acid precursor, which is processed to an eightresidue mature form containing a thio-lactone ring. Extracellular peptides bind to AgrC, triggering phosphorylation of AgrA, which activates expression of a gene encoding a noncoding RNA that, in turn, regulates a large number of pathogenesis determinants. Phospho-AgrA 


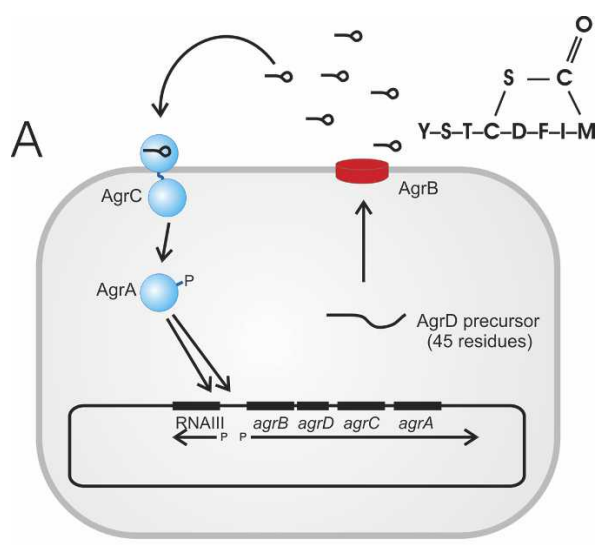

B

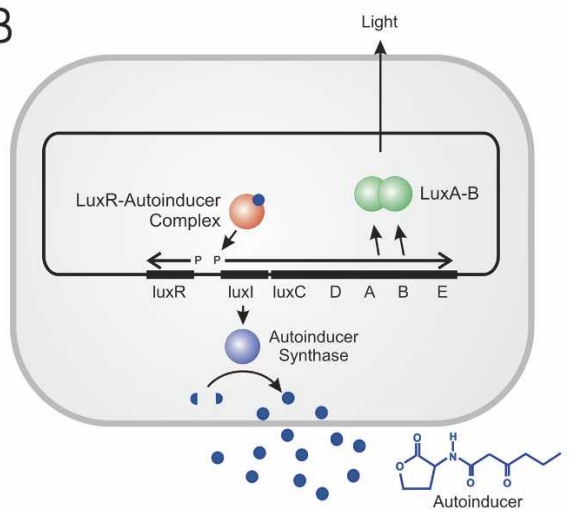

Figure 1. Two mechanisms of cell-cell signaling in bacteria. (A) Signaling via peptides by the pathogen S. aureus. (B) Signaling via acylhomoserine lactones by the marine bioluminescent bacterium $V$. fisheri.

also activates the agrBDCA operon, resulting in signal amplification. Homologous four-gene regulatory systems are found in many Gram-positive bacteria.

In other systems, peptide signals are imported by specific permeases and have cytoplasmic receptors. An example is found in the development of endospores by $\mathrm{Ba}$ cillus subtilis (Perego and Brannigan 2001). The 44amino-acid phrA product is processed to a linear pentapeptide and accumulates in the extracellular milieu. It is internalized by an oligopeptide-specific permease and inactivates the cytoplasmic RapA phosphatase. This allows the accumulation of phospho-Spo0F, which is required for sporulation and other stationary-phase activities. RapA is one of 11 homologous phosphatases, most or all of whose activities are regulated by cognate oligopeptides. Another bacterium, E. faecalis, also generates an extracellular oligopeptide signal from a larger precursor. This peptide is internalized via an oligopeptide permease and inactivates a repressor that controls genes required for conjugal transfer of DNA (Shi et al. 2005).

\section{Gram-negative proteo- and eubacteria}

Proteobacteria generally signal via acyl-homoserine lactones (AHLs), the archetypical quorum-sensing mol- ecules first discovered in Vibrio fisheri. AHLs are synthesized from S-adenosyl-L-methionine (SAM) and acylated acyl carrier protein (acyl-ACP) (Moré et al. 1996). The acyl chain length can vary from four to 18 carbons and can also vary in oxidation state and degree of desaturation (Fuqua et al. 1994). Most AHLs are made by proteins that resembles the LuxI protein of $V$. fisheri, and are detected by AHL-dependent transcription factors that resemble the $V$. fisheri LuxR protein. $V$. fischeri colonizes the light organs of various species of fish or marine invertebrates. As a population of $V$. fischeri cells grows within the light organ, the concentration of external AHL increases. When the autoinducer concentration reaches the micromolar range, its efflux from the cells becomes balanced by an influx, so that it can interact with LuxR. LuxR-autoinducer complexes bind the luciferase promoter and activate transcription (Fig. 1B).

The use of AHL-type autoinducers and proteins homologous to LuxI and LuxR extends to pathogenic bacteria that rely on these signals to colonize plants and animals. Probably the best studied example is Pseudomonas aeruginosa, which uses two LuxI/LuxR pairs (called LasI/LasR and RhlI/RhlR) that work in tandem to regulate expression of diverse virulence factors (Smith and Iglewski 2003). Hundreds of genes are induced by the two AHL signals, and dozens are repressed. The LasR/ LasI system is necessary for biofilm formation by $P$. aeruginosa, as lasI mutants make poorly differentiated biofilms that are easily disrupted. AHLs have also been detected in the sputum of cystic fibrosis (CF) patients, suggesting that quorum sensing could be vital for $P$. aeruginosa colonization of the CF lung.

A third type of chemical signal used for quorum sensing, called AutoInducer-2 (AI-2), was first discovered in Vibrio harveyi and is now known to be released by many groups of eubacteria (Waters and Bassler 2005). This raises the possibility that all these bacteria might use this molecule as a chemical "Esperanto" for intergeneric communication. AI- 2 is synthesized by a protein called LuxS, which is found in wide variety of bacteria. LuxS catalyzes a step in the turnover of SAM. When SAM is used as a methyl donor, S-adenosyl-homocysteine (SAH) is generated as one of the products. Removal of the adenosyl group by another enzyme generates S-ribosyl-homocysteine (SRH), which is converted by LuxS to homocysteine and 4,5-dihydroxy-2,3-pentanedione (DPD), which cyclizes to form AI-2. The AI-2 receptors of $V$. harveyi and Salmonella are periplasmic binding proteins called LuxP and LsrB, respectively. Unexpectedly, LuxP of $V$. harveyi contains an atom of boron adjacent to AI-2, while LsrB of Salmonella has a slightly modified form of AI-2 that lacks boron (Chen et al. 2002; Miller et al. 2004). LuxP of $V$. harveyi interacts with a two-component kinase, while LsrB of Salmonella is part of an AI-2 uptake system that leads to AI-2 catabolism.

Perhaps the most striking finding about AI- 2 is that it is synthesized by so many groups of bacteria and has such diverse roles in signaling. Pathogenic Escherichia coli strains mutated in luxS show decreased expression of a Type III protein translocation system. Mutations in 
lux also affect hemin acquisition genes in Porphyromonas gingivalis, the expression of the VirB virulence factor in Shigella flexneri, and the secretion of the SpeB cysteine protease virulence determinant of Streptococcus pyogenes. However, it is important to keep in mind that luxS mutants accumulate the precursor SRH, which could perturb cellular physiology and gene expression.

An interesting twist on the quorum-sensing phenomenon is that some bacteria have evolved mechanisms to destroy or block signaling molecules secreted by other bacteria or by host organisms. For example, an isolate of Bacillus produces an autoinducer inactivating enzyme called AiiA that hydrolyzes the lactone ring of AHLs, destroying their biological activity (Dong et al. 2001). Expression of AiiA by the plant pathogen Erwinia carotovora blocked quorum sensing and strongly attenuated virulence (Dong et al. 2000). Transgenic tobacco and potato plants expressing AiiA were almost fully resistant to E. carotovora. P. aeruginosa expressing AiiA was deficient in biofilm formation and showed attenuated pathogenicity. Humans and other mammals contain similar ring-opening enzymes in their sera (Yang et al. 2005). AI-2 can also be catabolized by Salmonella, thereby blocking AI-2-based quorum sensing by $V$. harveyi (Xavier and Bassler 2005). Another example of quorumsensing blocking is found in $S$. aureus, where signaling can be blocked by thiolactone peptides from heterologous strains (Ji et al. 1997).

\section{Quorum sensing: the yeast version}

In recent years, there have been several reports of quorum-sensing-like phenomena in fungal species. Intriguingly, each of these cases involves a morphological transition, from a filamentous, mycelial form to a yeast form, or vice versa. Whether this apparent theme represents the most common application of quorum sensing in eukaryotes, or more likely, the limited search for quorum sensing in eukaryotes, is not known. Presumably, these reports discussed below will spur a broader search for quorum sensing in fungi and other eukaryotes.

The regulation of the switch between the filamentous form and yeast form in the parasitic fungus Histoplasma capsulatum provided the first example of an apparent quorum-sensing mechanism in eukaryotes (Kügler et al. 2000). One home for this organism is the soil, where it exists as a free-living, saprophytic filamentous fungus. However, once humans (or other mammals) inhale aerosolized filament fragments, the growth habit switches to a yeast form, which invades and grows in macrophages. The yeast form produces some unique cell-wall polysaccharides [e.g., $\alpha$-(1,3)-glucan] in a cultured density-dependent fashion. This glucan is required for virulence. It is abundant in yeast cells growing in a dense culture, but most cells lose the glucan when diluted to a low density in fresh medium. However, if the fresh medium contains filtrate from a stationary-phase yeast culture, the cells continue to produce glucan. Together, these results suggest that yeast cells growing in a dense culture release a factor, perhaps akin to an autoinducer, that promotes glucan incorporation into the cell wall.

A similar density-dependent phenomenon has been reported for Ceratocystis ulmi, the dimorphic fungus that causes Dutch Elm disease. C. ulmi cells develop as budding yeast when inoculated at high density and as filamentous mycelia when inoculated at low density. The need for a large inoculum to promote growth as budding yeast could be bypassed if spent medium was added to the fresh medium, implying that growing cells excreted a quorum-sensing factor into the medium. The quorumsensing factor could be extracted from spent medium with an organic solvent, but its precise identity was not determined (Hornby et al. 2004).

Candida albicans, another dimorphic fungal human pathogen, relies on two quorum-sensing molecules to control its yeast-to-filamentous growth transition. The yeast-form cell develops a filamentous protrusion known as a germ tube at low cell densities, but cannot do so at high cell densities. One quorum-sensing molecule blocks this morphological transition at high cell density and has been identified as farnesol (Hornby et al. 2001). Farnesol is excreted continuously during growth of the culture in amounts proportional to cell density, consistent with the notion that it is a quorum-sensing molecule. In a separate study, the Fink laboratory (Chen et al. 2004) found a second quorum-sensing molecule, one that promotes the yeast-to-filamentous growth transition. When a dense culture is diluted into fresh medium at low density, there is a long lag phase before growth is resumed and the filamentous germ tube is produced. This lag can be shortened by the addition of conditioned medium. The active molecule in conditioned medium was purified and identified as tyrosol. Addition of tyrosol alone to dilute cultures both shortened the lag before growth resumption and induced germ tube formation. With an identified quorum-sensing molecule in hand, Chen et al. (2004) used genome expression profiling to compare transcript levels of cells grown at high density and cells grown at low density with and without tyrosol. The genes that showed the greatest reduction after dilution, but no reduction when tyrosol was present, encode proteins involved in DNA synthesis and cell cycle regulation. This finding both rationalizes the long lag in growth and shows that, as in bacterial quorum sensing, C. albicans quorum sensing influences transcription.

Together, these investigations of quorum sensing in $C$. albicans present a provocative picture. One quorumsensing molecule, farnesol, inhibits formation of germ tubes when cultures reach high density. A second quorum-sensing molecule, tyrosol, promotes cell growth and the formation of germ tubes at low density. Unraveling the mechanisms that underlie this reciprocal control of germ tube formation and the metabolic regulation that operates in response to these environmental cues is a fascinating challenge for the future.

The examples of apparent quorum sensing in fungal species illustrate one element of the quorum-sensing phenomenon in bacteria: a density-dependent effect on cell physiology. However, in these studies, the other 
hallmarks of bacterial quorum sensing_an identified signal transduction pathway and transcription factors governing the physiological response-were not described. Hence, the degree of similarity between bacterial and fungal quorum-sensing processes was not clear. Those gaps have now been filled by an exciting set of findings from the Fink laboratory published in this issue of Genes \& Development (Chen and Fink 2006).

Like the fungal species described thus far, the wellstudied budding yeast, $S$. cerevisiae, can undergo morphological transition from the yeast form to a filamentous form in response to environmental cues. One cue that triggers this transition is nitrogen-poor growth medium, a condition that activates several distinct signal transduction pathways. One of these pathways is the Ras-cAMP-dependent protein kinase (PKA) pathway, a well-known pathway, but one whose mechanism of activation has remained obscure. In their report, Chen and Fink (2006) not only establish the role of quorum sensing in filamentous growth, but also provide some intriguing molecular clues to PKA signaling.

The starting point for the Chen and Fink (2006) study was the observation that conditioned medium from $S$. cerevisiae stationary-phase cultures strongly induces filamentous growth. At least in part, this induction is a consequence of the fivefold stimulation of transcription of FLO11, a gene essential for filamentous growth. The active molecules were purified and shown to be phenylethanol and tryptophol, two aromatic alcohols derived from phenylalanine and trytophan, respectively. The addition of both alcohols causes a more vigorous filamentous growth response and induction of FLO11 than does either singly, implying some synergy in their action. Based on the bacterial paradigm, these molecules have the features one expects for a quorum-sensing molecule. First, their production is growth-phase-dependent, accumulating to the highest level when cultures enter stationary phase. Second, the production of tryptophol (but not phenylethanol) is autostimulatory. The addition of tryptophol to cells dramatically induces the expression of genes (ARO9 and $A R O 10)$ required to convert tryptophan to tryptophol. Third, mutants unable to produce these alcohols show greatly diminished filamentous growth and FLO11 expression.

The identification of phenylethanol and tryptophol as quorum-sensing molecules for filamentous growth also provides links to known signaling and regulatory mechanisms that influence morphogenesis. One link is to nitrogen regulation. Ammonium ion is known to repress filamentous growth, and it also represses transcription of ARO9 and ARO10, the two genes required for aromatic alcohol production. The second link is to the cAMPPKA pathway and its target transcription factor Flo8, both of which are known to be required for filamentous growth. The effect of the aromatic alcohols requires a particular cAMP protein kinase, Tpk2, and the Flo8 transcription factor. A second signal transduction pathway required for filamentous growth, a mitogen-activate protein (MAP) kinase-based pathway, is not required for transcription induction of FLO11 by aromatic alcohols, raising the possibility that these alcohols directly regulate signaling in the Ras-PKA pathway.

Together, these experiments provide a compelling case for the role of quorum sensing in filamentous growth by yeast, a case as mature, complete, and thorough as the one for quorum sensing in bacteria. The experiments also raise many fascinating questions for the future. What are the direct targets of the aromatic alcohols, the cAMP-dependent protein kinase Tpk2 itself, or some other protein? How common is quorum sensing among eukaryotic organisms? Is it limited to fungi, or distributed more widely? The fact that four different organic alcohols have been found as quorum-sensing molecules suggests that there is evolutionary diversity in the underlying mechanisms. If so, a reasonable corollary is that additional quorum-sensing processes, perhaps a large number, await discovery.

Thus far, the quorum-sensing phenomena described in fungi share a theme: They all involve morphogenetic transitions. Might quorum sensing be found to regulate other physiological transitions as well? The Chen and Fink (2006) study concludes with a glimpse of what might be found. In particular, they show by whole-genome microarray expression analysis that the aromatic alcohols regulate the transcription of a small set of genes, $\sim 150$. Strikingly, $\sim 70 \%$ of these genes have previously been shown to be up-regulated upon entry into stationary phase. The induction of these genes suggests that quorum-sensing molecules may also play a role in signaling the transition to the stationary phase. Perhaps entry into stationary phase will be a fruitful setting for searching for quorum sensing in other organisms.

\section{References}

Chen, H. and Fink, G.R. 2006. Feedback control of morphogenesis in fungi by aromatic alcohols. Genes \& Dev. (this issue).

Chen, X., Schauder, S., Potier, N., Van Dorsselaer, A., Pelczer, I., Bassler, B.L., and Hughson, F.M. 2002. Structural identification of a bacterial quorum-sensing signal containing boron. Nature 415: 545-549.

Chen, H., Fujita, M., Feng, Q., Clardy, J., and Fink, G.R. 2004 Tyrosol is a quorum-sensing molecule in Candida albicans. Proc. Nat1. Acad. Sci. 101: 5048-5052.

Dong, Y.H., Xu, J.L., Li, X.Z., and Zhang, L.H. 2000. AiiA, an enzyme that inactivates the acylhomoserine lactone quorum-sensing signal and attenuates the virulence of Erwinia carotovora. Proc. Natl. Acad. Sci. 97: 3526-3531.

Dong, Y.H., Wang, L.H., Xu, J.L., Zhang, H.B., Zhang, X.F., and Zhang, L.H. 2001. Quenching quorum-sensing dependent bacterial infection by an $\mathrm{N}$-acyl homoserine lactonase. $\mathrm{Na}$ ture 411: 813-817.

Dunny, G.M. and Leonard, B.A. 1997. Cell-cell communication in gram-positive bacteria. Annu. Rev. Microbiol. 51: 527564.

Fuqua, W.C., Winans, S.C., and Greenberg, E.P. 1994. Quorum sensing in bacteria: The LuxR-LuxI family of cell densityresponsive transcriptional regulators. J. Bacteriol. 176: 269275 .

Hornby, J.M., Jensen, E.C., Lisec, A.D., Tasto, J.J., Jahnke, B., Shoemaker, R., Dussault, P., and Nickerson, K.W. 2001. Quorum sensing in the dimorphic fungus Candida albicans 
is mediated by farnesol. Appl. Environ. Microbiol. 67: 29822992.

Hornby, J.N.M., Jacobitz-Kizzir, S.M., McNeel, D.J., Jensen, E.C., Treves, D.S., and Nickerson, K.W. 2004. Inoculum size effect in dimorphic fungi: Extracellular control of yeast-mycelium dimorphism in Ceratocystis ulmi. Appl. Environ. Microbiol. 70: 1356-1359.

Ji, G., Beavis, R., and Novick, R.P. 1997. Bacterial interference caused by autoinducing peptide variants. Science 276: 20272030.

Kügler, S., Sebghati, T.C., Eissenberg, L.G., and Goldman, W.E. 2000. Phenotypic variation and intracellular parasitism by Histoplasma capsulatum. Proc. Natl. Acad. Sci. 97: 87948798.

Lyon, G.J. and Novick, R.P. 2004. Peptide signaling in Staphylococcus aureus and other Gram-positive bacteria. Peptides 25: 1389-1403.

Miller, S.T., Xavier, K.B., Campagna, S.R., Taga, M.E., Semmelhack, M.F., Bassler, B.L., and Hughson, F.M. 2004. Salmonella typhimurium recognizes a chemically distinct form of the bacterial quorum-sensing signal AI-2. Mol. Cell 15: 677687.

Moré, M.I., Finger, L.D., Stryker, J.L., Fuqua, C., Eberhard, A., and Winans, S.C. 1996. Enzymatic synthesis of a quorumsensing autoinducer through use of defined substrates. Science 272: 1655-1658.

Natsume, R., Ohnishi, Y., Senda, T., and Horinouchi, S. 2004. Crystal structure of a $\gamma$-butyrolactone autoregulator receptor protein in Streptomyces coelicolor A3(2). J. Mol. Biol. 336: 409-419.

Pappas, K.M., Weingart, C.L., and Winans, S.C. 2004. Chemical communication in proteobacteria: Biochemical and structural studies of signal synthases and receptors required for intercellular signalling. Mol. Microbiol. 53: 755-769.

Perego, M. and Brannigan, J.A. 2001. Pentapeptide regulation of aspartyl-phosphate phosphatases. Peptides 22: 1541-1547.

Qazi, S.N.A., Counil, E., Morrissey, J., Rees, C.E.D., Cockayne, A., Winzer, K., Chan, W.C., Williams, P., and Hill, P.J. 2001. agr expression precedes escape of internalized Staphylococcus aureus from the host endosome. Infect. Immun. 69: 7074-7082.

Redfield, R.J. 2001. Is quorum sensing a side effect of diffusion sensing? Trends Microbiol. 10: 365-370.

Shi, K., Brown, C.K., Gu, Z.Y., Kozlowicz, B.K., Dunny, G.M., Ohlendorf, D.H., and Earhart, C.A. 2005. Structure of peptide sex pheromone receptor $\operatorname{PrgX}$ and $\operatorname{PrgX} /$ pheromone complexes and regulation of conjugation in Enterococcus faecalis. Proc. Natl. Acad. Sci. 102: 18596-18601.

Smith, R.S. and Iglewski, B.H. 2003. P. aeruginosa quorumsensing systems and virulence. Curr. Opin. Microbiol. 6: 5660.

Waters, C.M. and Bassler, B.L. 2005. Quorum sensing: Cell-tocell communication in bacteria. Annu. Rev. Cell Dev. Biol. 21: 319-346.

Whitehead, N.A., Barnard, A.M., Slater, H., Simpson, N.J., and Salmond, G.P. 2001. Quorum-sensing in Gram negative bacteria. FEMS Microbiol. Rev. 25: 365-404.

Xavier, K.B. and Bassler, B. 2005. Interference with AI-2-mediated bacterial cell-cell communication. Nature 437: 750753.

Yang, F., Wang, L.H., Wang, J., Dong, Y.H., Hu, J.Y., and Zhang, L.H. 2005. Quorum quenching enzyme activity is widely conserved in the sera of mammalian species. FEBS Lett. 579: 3713-3717. 


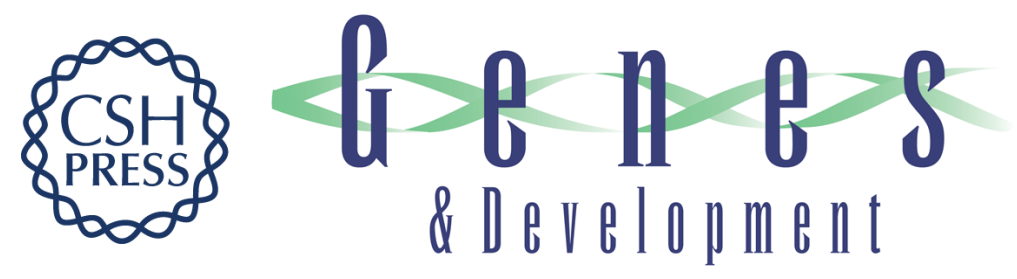

\section{Eukaryotes learn how to count: quorum sensing by yeast}

George F. Sprague, Jr. and Stephen C. Winans

Genes Dev. 2006, 20:

Access the most recent version at doi:10.1101/gad.1432906

Related Content Feedback control of morphogenesis in fungi by aromatic alcohols Hao Chen and Gerald R. Fink

Genes Dev. May , 2006 20: 1150-1161

References This article cites 24 articles, 9 of which can be accessed free at:

http://genesdev.cshlp.org/content/20/9/1045.full.html\#ref-list-1

Articles cited in:

http://genesdev.cshlp.org/content/20/9/1045.full.html\#related-urls

\section{License}

Email Alerting

Service

Receive free email alerts when new articles cite this article - sign up in the box at the top right corner of the article or click here.

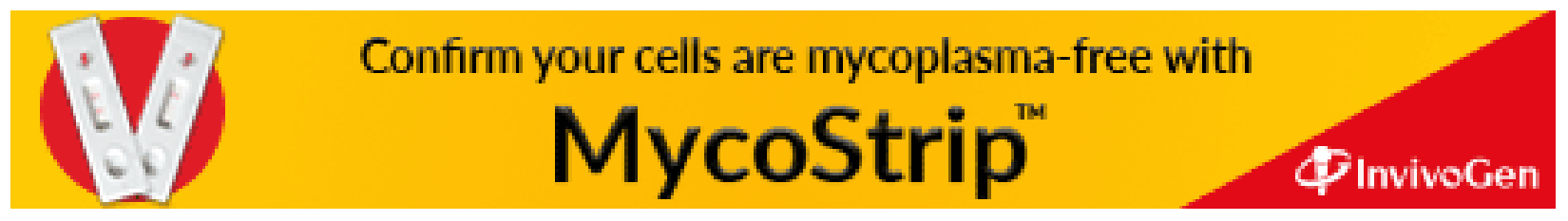

